\title{
Authors' reply re: Implications for the future of Obstetrics and Gynaecology following the COVID-19 pandemic: A commentary. (Response to BJOG-20-1954)
}

\author{
Lorraine Kasaven $^{1}$, Srdjan Saso ${ }^{2}$, Jennifer Barcroft ${ }^{1}$, Joseph Yazbek ${ }^{2}$, Karen Joash ${ }^{2}$, \\ Catriona Stalder ${ }^{3}$, Jara Ben-Nagi ${ }^{4}$, J Richard Smith ${ }^{5}$, Christoph Lees ${ }^{1}$, Tom Bourne ${ }^{1}$, and \\ Benjamin Jones ${ }^{2}$ \\ ${ }^{1}$ Imperial College Healthcare NHS Trust \\ ${ }^{2}$ Imperial College London \\ ${ }^{3}$ Queen Charlotte's and Chelsea Hospital \\ ${ }^{4}$ The Centre for Reproductive and Genetic Health (CRGH) \\ ${ }^{5}$ Hammersmith Hospital
}

October 6, 2020

Dear Sir,

Whilst the primary focus of our commentary was to reflect upon the multitude of clinical and institutional changes prompted by COVID-19 to help adopt a more streamlined approach to healthcare, ${ }^{1}$ we thank Herron et al for highlighting the importance of partner support during labour. ${ }^{2}$ However, we note that even during the peak of the first wave of infections, the Royal College of Obstetricians and Gynaecologists (RCOG) continued to advocate the presence of a single birth partner throughout labour. Many obstetric units, including our own, managed to successfully adhere to this practice throughout the pandemic. However, guidance from the RCOG for women attending antenatally, for face to face clinic appointments or ultrasound scans, was to attend alone. This was subsequently implemented in most hospitals in order to reduce the number of visitors. ${ }^{1}$ Whilst necessary during the initial fear and uncertainty surrounding COVID-19, moving forward it is important to consider the potential negative impact of partner non-attendance antenatally, as well as intrapartum. Partners often positively encourage women to seek care and prepare for birth complications, thereby preventing delay in treatment and helping to manage expectations, which have been shown to positively impact outcomes. ${ }^{3}$ Whilst undoubtedly an exciting time for many, pregnancy and the prospect of motherhood is daunting to others. Partners provide support and facilitate decision making throughout the antenatal process, particularly in difficult circumstances such as following the diagnosis of a missed miscarriage, during counselling for pregnancies affected by genetic abnormalities, or after an intrauterine death. The restrictions on partner attendance may therefore inadvertently prevent a number of women seeking care during pregnancy, for fear of having to face procedures or receiving bad news alone. Evidence from a London hospital supports this notion after demonstrating a significant increase in stillbirth rate during the pandemic compared to pre-pandemic (9.31 per 1000 births Vs 2.38 per 1000 births; $p=0.01$ ). Of significance, no cases were affected by COVID-19, nor were there any post-mortem findings suggestive of the virus. $^{4}$

The utilisation of remote consultations with a woman and her partner offers a suitable option in appropriately triaged cases. ${ }^{1}$ Even in remote consultations where inadvertent difficult decisions arise, the presence and support of their partner facilitates collaborative decision making. Ironically, those with high risk enough pregnancies to warrant in person consultations, where additional support could offer significant value, are 
those whereby partners are not permitted. Prior to the pandemic, partners often reported feeling excluded, fearful of the uncertainty of pregnancy and labour and frustrated by perceived lack of support from healthcare professionals. ${ }^{5}$ This may subsequently negatively impact their relationship because of the inability to adequately support their partners. Their exclusion from the majority of antenatal care therefore, may not only negatively impact psychological wellbeing of women which may in turn result in suboptimal outcomes, but also negatively impact their future relationship. As such, we agree with Herron et al and support their notion that attempts should be made towards delivering individualised patient centred care both antenatally and intrapartum.

Lorraine S Kasaven ${ }^{1,2}$, Srdjan Saso ${ }^{1,2}$, Jen Barcroft ${ }^{1,2}$, Joseph Yazbek ${ }^{1,2}$, Karen Joash ${ }^{1}$, Catriona Stalder ${ }^{1}$, Jara Ben Nagi, ${ }^{2}$ J Richard Smith, ${ }^{1,2}$ Christoph Lees ${ }^{1,2}$, Tom Bourne ${ }^{1,2}$, Benjamin P Jones ${ }^{1,2}$

${ }^{1}$ Queen Charlotte's and Chelsea Hospital, Department of Cancer and Surgery, Imperial College NHS Trust, W12 0HS London, UK.

${ }^{2}$ Imperial College London, Department of Cancer and Surgery, London W12 0NN, UK.

\section{References:}

(1) Kasaven LS, Saso S, Barcroft J, Yazbek J, Joash K, Stalder C, et al. Implications for the future of Obstetrics and Gynaecology following the COVID-19 pandemic: a commentary. BJOG: An International Journal of Obstetrics \& Gynaecology 2020. Oct;127(11):1318-1323. doi: 10.1111/1471-0528.16431. Epub 2020 Aug 12. PMID: 32716588.

(2) Herron J, Herron R. Re: Implications for the future of Obstetrics and Gynaecology following the COVID19 pandemic: A commentary. (First comment letter. Reference to be added).

(3) Odimegwu C, Adewuyi A, Odebiyi T, Aina B, Adesina Y, Olatubara O, et al. Men's role in emergency obstetric care in Osun State of Nigeria. Afr J Reprod Health 2005 Dec;9(3):59-71. PMID: 16623190.

(4) Khalil A, Von Dadelszen P, Draycott T, Ugwumadu A, O'Brien P, Magee L. Change in the incidence of stillbirth and preterm delivery during the COVID-19 pandemic. JAMA. 2020 Jul 10;324(7):705-6. doi: 10.1001/jama.2020.12746. Epub ahead of print. PMID: 32648892; PMCID: PMC7435343.

(5) Steen M, Downe S, Bamford N, Edozien L. Not-patient and not-visitor: a metasynthesis fathers' encounters with pregnancy, birth and maternity care. Midwifery. 2012 Aug;28(4):362-71. doi: 10.1016/j.midw.2011.06.009. Epub 2011 Aug 6. PMID: 21820778. 